\title{
Adaptación fonológica de préstamos léxicos del español en el noruego
}

\author{
Phonological adaptation of lexical borrowings from Spanish in Norwegian
}

\author{
HAAKON S. KROHN \\ Escuela de Filología, Lingüística y Literatura \\ Universidad de Costa Rica, Costa Rica
}

\section{Resumen}

En este artículo se lleva a cabo un análisis de la adaptación fonológica de 76 préstamos léxicos del español en el noruego. Las adaptaciones se interpretan de acuerdo con la descripción generativa del sistema fonológico noruego de Kristoffersen (2000). A nivel segmental, se observa una fuerte influencia de la ortografía, lo cual indica que este factor no debe subestimarse en estudios de la misma índole. Sin embargo, también hay ciertas adaptaciones de fonemas que solo pueden ser explicadas por influencia fonológica o fonética del español. A nivel suprasegmental, se mantiene la posición del acento léxico del español en la mayoría de los préstamos, y casi todos son asignados el tonema 1, que se manifiesta como el no marcado.

Palabras clave: fonología, préstamos léxicos, noruego, español

\section{Abstract}

In this paper we carry out an analysis of the phonological adaptation of 76 lexical borrowings from Spanish in Norwegian. The adaptations are interpreted according to Kristoffersen's (2000) generative description of the Norwegian phonological system. On the segmental level, a strong influence of the orthography is observed, which indicates that this factor should not be underestimated in studies of the same nature. However, there are also certain phoneme adaptations that can only be explained as a result of phonological or phonetic influence from Spanish. On the suprasegmental level, the position of the lexical stress in Spanish is retained in the majority of the borrowings, and almost all of them are assigned toneme 1 , which manifests itself as the unmarked one.

Keywords: phonology, lexical borrowings, Norwegian, Spanish 


\section{Introducción}

$\mathrm{E}$ l vocabulario del noruego ${ }^{1}$ abarca una cantidad considerable de préstamos del español: 94 de las palabras en el diccionario oficial del noruego bokmål, ${ }^{2}$ Bokmålsordboka (Universitetet i Bergen y Språkrådet, 2020), están etiquetadas como provenientes del español. Todas estas han sido adaptadas al sistema fonológico noruego, a tal grado que ninguna se pronuncia con sonidos ajenos del noruego y todas están integradas en el sistema prosódico de este idioma. En el presente artículo se analizan los diferentes patrones de adaptación fonológica de 76 de estos préstamos, tanto a nivel segmental como suprasegmental. Es la primera investigación que se centra en la adaptación fonológica de préstamos entre estos dos idiomas en específico.

Primeramente, se elaboró una lista de préstamos léxicos del español consolidados en el noruego. Estos se transcribieron tanto fonológica como fonéticamente, con base en el dialecto noruego hablado por el investigador, una variedad muy cercana a la que a menudo es denominada standard østnorsk ("noruego oriental estándar"). A partir de estos datos, se indagó en las correspondencias de cada uno de los fonemas españoles con los del noruego, así como en la posición del acento léxico y la asignación de tonema. El fundamento teórico del análisis es el modelo generativo presentado por Kristoffersen (2000) para el sistema fonológico del noruego.

\section{Estado de la cuestión}

Nunca se había estudiado específicamente la integración fonológica de los préstamos del español en el noruego, pero sí se habían realizado indagaciones de esta índole de palabras noruegas provenientes del inglés. Lea (2009, p. 63) señala que la pronunciación de los anglicismos varía mucho, según el nivel de dominio que el hablante tenga del inglés y el tiempo que el préstamo haya sido utilizado en el noruego; en varios casos, se emplean sonidos originalmente inexistentes en el noruego.

En cuanto a las consonantes inglesas $/ \theta /$ y / //, tradicionalmente han sido sustituidas en noruego por $/ \mathrm{t} / \mathrm{y} / \mathrm{d} /$, respectivamente, pero en el corpus estudiado por Lea (2009) tienden a pronunciarse $[\theta]$ y [ð], o bien, se eliden (p. 64). De manera similar, el fonema inglés $/ \mathrm{w} /$ se ha sustituido por /o/ en los préstamos más antiguos, mientras que Lea (2009, p. 64) nota que los préstamos más recientes se pronuncian con [w]. En cambio, la consonante/z/ todavía es reemplazada por/s/ en noruego, mientras que la vocal $/ \Lambda /$ es sustituida por /ø/ (Lea, 2009, p. 65). Tanto Brandsegg (2001, pp. 100-101) como Lea (2009, p. 65) notan que la adaptación de /./ varía; en algunas ocasiones se mantiene la pronunciación inglesa [x], y en otras es sustituida por [r]. Con respecto a la entonación, Andersen (2007, pp. 7576) y Lea (2009, p. 64) concuerdan en que los anglicismos se pronuncian con entonación noruega.

En lo que concierne a los préstamos de otras lenguas, la situación parece ser distinta. Con base en observaciones en un corpus oral, Lea $(2009$, p. 66) afirma que "préstamos de otras lenguas que el inglés se pronuncian con sonidos que ya existen en la fonología noruega". ${ }^{3}$

Por otro lado, hay muchos estudios de la integración de préstamos del español en otras lenguas. Sin embargo, 
se trata de lenguas habladas en países mayoritariamente hispanohablantes o en comunidades lingüísticas con alto grado de contacto con el español. Algunos ejemplos son el náhuatl (San Giacomo y Peperkamp, 2008), el quechua (Feke, 2003), el guaraní (Pinta, 2013), el vasco (Hualde, 2009) y el árabe marroquí septentrional (Sayahi, 2005). Por lo tanto, la adaptación de préstamos del español en estas lenguas tiene lugar bajo condiciones muy distintas que en el noruego, ya que, indudablemente, existe menor conocimiento acerca de la fonología y la fonética del español en Noruega.

\section{Marco teórico}

\section{Influencia fonética, fonológica u ortográfica}

En las teorizaciones acerca de la adaptación de préstamos léxicos, generalmente se identifican dos puntos de vista contrastivos (Chang, 2008, p. 43): el énfasis en la fonética y el énfasis en la fonología. Un representante del punto de vista fonético es Silverman (1992), quien afirma que los fonos de la lengua fuente son vinculados con los fonemas de la lengua meta con base en similitud fonética y que, en un segundo paso, las restricciones fonológicas de la lengua meta se imponen sobre esta representación. Esto quiere decir que dos alófonos de un mismo fonema de la lengua fuente pueden ser reinterpretados como dos fonemas distintos en la lengua meta. Peperkamp (2005), por su lado, sostiene que los préstamos ni siquiera llegan a formar parte de la gramática fonológica de la lengua fuente, por lo que todas las transformaciones son fonéticas. En cambio, LaCharité y Paradis (2005) abogan por una perspectiva según la cual los hablantes bilingües son los responsables de introducir los préstamos en una lengua; de esta manera, los segmentos de la lengua fuente son reemplazados por el segmento fonológicamente más semejante en la lengua meta. Otros estudios, como el de Chang (2008), toman una posición intermedia y sugieren que las adaptaciones son influenciadas tanto por los detalles fonéticos como por los sistemas fonológicos de las lenguas en cuestión.

Calabrese y Wetzels (2009, p. 1-2) señalan que los préstamos ocurren en dos tipos de escenarios: pueden ser implementados por personas con competencia tanto en la lengua fuente como en la lengua meta, o bien, por personas que tienen bajo dominio de la lengua fuente o que la desconocen por completo. El primer caso es etiquetado por los autores como "nativización por medio de producción”, mientras que el segundo lo llaman "nativización por medio de percepción”. Solo en la segunda situación, la adaptación puede basarse únicamente en la señal acústica percibida (Calabrese y Wetzels, 2009, p. 3), puesto que el bilingüismo implica conocimiento de los sistemas fonológicos de ambos idiomas. Por otro lado, para el primer escenario se requieren hablantes bilingües.

El caso de los préstamos del español en el noruego corresponde probablemente, en mayor grado, a la "nativización por medio de percepción", puesto que el único idioma no escandinavo conocido extensamente en Noruega como segunda lengua es el inglés. Sin embargo, no existen datos acerca de la cantidad de hispanohablantes en 
Noruega, por lo que es imposible determinar cuántos hablantes bilingües de noruego y español existen. De todos modos, durante las últimas décadas, el español se ha convertido en el segundo idioma extranjero más estudiado en la educación secundaria (ungdomsskolen, correspondiente a los años 8-10 de la carrera educativa): el 46 por ciento de los alumnos que matricularon un curso de un idioma extranjero aparte del inglés en el 2018 escogieron español (Foss, 2019). El español se ha mantenido en esta posición desde el 2011, que es el primer año del que existen estadísticas disponibles.

Un factor adicional en la adaptación de préstamos, que ha sido poco considerado en estudios anteriores, es la influencia de la ortografía. Como señalan Vendelin y Peperkamp (2006, p. 997), los investigadores que sí toman en cuenta este factor suelen considerarlo marginal. No obstante, la indagación de dichas lingüistas sugiere una influencia importante en el proceso de adaptación de préstamos. Además, afirman (p. 1004) que las adaptaciones basadas en la forma ortográfica de las palabras en la lengua fuente muchas veces son indistinguibles de las adaptaciones basadas en minimidad fonológica o fonética, por lo que la influencia de la ortografía necesariamente es subestimada. De acuerdo con ellas (p. 997), la ortografía puede influir en la adaptación de los préstamos de dos maneras. En primer lugar, algunos préstamos son "adaptaciones de lectura", lo cual quiere decir que se pronuncian como si fueran palabras nativas en la lengua meta. En segundo lugar, puede existir cierto grado de estandarización respecto a cómo debe pronunciarse cada grafema de la lengua fuente, incluso para los grafemas que en realidad representan varios fonemas distintos.

\section{Sistema fonológico del noruego}

En este apartado se presenta una síntesis del sistema fonológico del noruego. El fundamento teórico es el modelo generativo de Kristoffersen (2000), la descripción fonológica más detallada que ha sido publicada de este idioma. Es desarrollada dentro del marco de la Fonología Léxica y trata una variedad del noruego comúnmente conocida como standard østnorsk ("noruego oriental estándar"), hablada por gran parte de la población en el sureste de Noruega, incluyendo la capital Oslo. Sin embargo, a pesar de la inclusión de la palabra "estándar" en la denominación, la variedad nunca ha sido oficialmente estandarizada. De hecho, mientras el noruego cuenta con dos normas ortográficas oficiales -bokmål y nynorsk-, no existe ninguna norma reconocida para la pronunciación (cf. Kristoffersen, 2000, p. 6-7). Como resultado, los noruegohablantes tienden a apegarse a la pronunciación tradicional de su dialecto local en cualquier situación, por lo que también existe una variación notable dentro de lo que se suele considerar standard østnorsk. Con el fin de restringir el objeto de estudio, todas las pronunciaciones noruegas analizadas en el presente artículo representan el habla del investigador, proveniente de la zona costera de Vestfold en el Fiordo de Oslo, la cual puede considerarse una variedad específica del standard østnorsk. De aquí en adelante, todas las referencias al "noruego" aludirán solamente a esta variedad lingüística en particular. 


\section{Vocales}

El sistema vocálico del noruego se diferencia del sistema del español principalmente por el hecho de distinguir entre un mayor número de calidades vocálicas: nueve en el noruego versus cinco en el español. Las vocales noruegas suelen ser representadas por los siguientes símbolos: /i/, /y/, /u/, /u/, /e/, /ø/, /o/, /æ/ y /a/. Según Kristoffersen (2000, p. 14), el fonema /æ/ es marginal, debido a que aparece en distribución complementaria con /e/ en la mayoría de los casos: [æ] solo ocurre ante $/ \mathrm{f} /, \mathrm{j} /$, $/ \mathrm{v} /$ y consonantes retroflejas, mientras que los alófonos de /e/ generalmente no se observan en dichos entornos. Sin embargo, sí existe una cantidad considerable de pares mínimos que contras$\tan / æ / \mathrm{y} / \mathrm{e} /$ ante $/ \mathrm{r} /$.

Además de las calidades vocálicas, en el noruego existe una diferenciación entre vocales cortas y largas, que tradicionalmente se ha considerado fonológica (cf. Torp y Vikør, 2000, p. 56; Endresen, 1991, p. 183). Empero, de acuerdo con el análisis de Kristoffersen (2000), la duración vocálica es un fenómeno fonético determinado por el acento léxico y la moracidad de la consonante siguiente. En sílabas inacentuadas (es decir, sin acento léxico primario), las vocales siempre se pronuncian como cortas. Las sílabas acentuadas, por su lado, siempre se realizan como "pesadas", lo cual quiere decir que su rima tiene que consistir en una vocal corta seguida de al menos una consonante moraica, o bien, en una vocal larga sin consonante moraica (Kristoffersen, 2000, p. 166).
Queda claro, entonces, que el peso silábico es representado por medio de moras: las sílabas ligeras consisten en una mora y las pesadas, en dos. Una vocal corta es monomoraica, una vocal larga es bimoraica, mientras que una consonante puede o no ser moraica. Dicha propiedad de las consonantes está codificada fonológicamente y es justamente esta la que determina la duración de una vocal: en las sílabas acentuadas, la vocal es corta si le sigue una consonante moraica, y larga en el caso contrario. Las realizaciones fonéticas de las consonantes moraicas se explican con mayor detalle más adelante.

En la tabla 1 se presentan las calidades vocálicas distintivas del noruego, junto con una descripción aproximada de su realización en sílabas acentuadas, sus alófonos (en sílabas tanto inacentuadas como acentuadas) y su representación ortográfica. Aquí se puede observar, en primer lugar, que las vocales medias (/e/, /ø/ y /o/) se cierran ligeramente cuando se realizan como largas. Además, la variedad corta de /e/ es generalmente [ $\varepsilon]$, pero suele ser [ə] en sílabas inacentuadas con ataque silábico que no sean la primera sílaba de la palabra. Al igual que en Kristoffersen (2000), se emplean los símbolos [i, y, u, u] para las vocales cerradas cortas, aunque también podrían transcribirse [I, Y,,,$\mho]$ ]. En cuanto a los grafemas, se consigna primero el que más comúnmente se asocia con el fonema en cuestión y luego, entre paréntesis, otros grafemas utilizados para el representar el mismo fonema en casos más específicos. 
Tabla 1

Fonemas vocálicos del noruego

\begin{tabular}{|c|c|c|c|}
\hline Fonema & Descripción & Realizaciones & Repr. ortográfica \\
\hline /i/ & $\begin{array}{l}\text { alta, anterior, } \\
\text { no redondeada }\end{array}$ & {$[\mathrm{i}] \sim[\mathrm{i}:]$} & $<\mathrm{i}>$ \\
\hline$/ y /$ & $\begin{array}{l}\text { alta, anterior, } \\
\text { redondeada }\end{array}$ & {$[\mathrm{y}] \sim[\mathrm{y}:]$} & $<y>$ \\
\hline$/ \mathfrak{u} /$ & $\begin{array}{l}\text { alta, central, } \\
\text { redondeada }\end{array}$ & {$[\mathrm{H}] \sim[\mathrm{H}:]$} & $<\mathrm{u}>$ \\
\hline$/ \mathrm{u} /$ & $\begin{array}{l}\text { alta, posterior, } \\
\text { redondeada }\end{array}$ & {$[\mathrm{u}] \sim[\mathrm{u}:]$} & $<0>(<\mathrm{u}>)$ \\
\hline /e/ & $\begin{array}{c}\text { media, anterior, } \\
\text { no redondeada }\end{array}$ & {$[\varepsilon] \sim[ə] \sim[\mathrm{e}:]$} & $<\mathrm{e}>(<\Re>)$ \\
\hline $\mid \varnothing /$ & $\begin{array}{l}\text { media, central, } \\
\text { redondeada }\end{array}$ & {$[œ] \sim[\varnothing:]$} & $<\varnothing>$ \\
\hline /o/ & $\begin{array}{l}\text { media, posterior, } \\
\text { redondeada }\end{array}$ & {$[0] \sim[0:]$} & $<\stackrel{\mathrm{a}}{>}(<0>)$ \\
\hline /æ/ & $\begin{array}{l}\text { baja, central, } \\
\text { no redondeada }\end{array}$ & {$[æ] \sim[æ:]$} & $\begin{array}{c}<æ> \\
(<\mathrm{e}>,<\mathrm{a}>)\end{array}$ \\
\hline /a/ & $\begin{array}{l}\text { baja, posterior, } \\
\text { no redondeada }\end{array}$ & {$[a] \sim[a:]$} & $<\mathrm{a}>$ \\
\hline
\end{tabular}

Fuente: Kristoffersen (2000, pp. 13-21).

La representación ortográfica de las vocales también es de gran relevancia para el análisis de la adaptación de los préstamos del español, como quedará claro más adelante. A este respecto, se puede decir que a cada grafema vocálico le corresponde canónicamente un fonema, equivalente al nombre de la letra. Cuando la vocal es larga, casi siempre se cumple con la correspondencia entre el grafema y su fonema canónico. En cambio, cuando la vocal se realiza como corta, el grafema muchas veces no es el canónico, lo cual es el resultado de una fosilización ortográfica. En la tabla 2 se muestran los fonemas canónicamente asociados con cada grafema, así como los otros fonemas que los grafemas pueden representar. 
Tabla 2

Fonemas asociados con cada grafema vocálico en el noruego

\begin{tabular}{|c|c|c|}
\hline Grafema & Fonema canónico & Otros fonemas \\
\hline$<\mathrm{a}>$ & $/ a /$ & /æ/ en el diptongo /æv/ (<au>) \\
\hline$<\mathrm{e}>$ & /e/ & $\begin{array}{c}/ æ / \text { ante }<\mathrm{r}>\text { (pero con varias excepciones), y en el } \\
\text { diptongo /æj/ }(<\mathrm{ei}>)\end{array}$ \\
\hline$<\mathrm{i}>$ & /i/ & $\begin{array}{c}\text { /j/ en los diptongos /aj/ }(<\mathrm{ai}>), / æ \mathrm{jj} /(<\mathrm{ei}>), / \mathrm{oj} /(<\mathrm{oi}>) \text { y } \\
/ \mathrm{uj} /(<\mathrm{ui}>)\end{array}$ \\
\hline$<0>$ & $/ \mathrm{u} /$ & $\begin{array}{l}\text { /o/ en muchas sílabas cerradas, y en sílabas abiertas } \\
\text { ante }<\mathrm{v}>\end{array}$ \\
\hline$<\mathrm{u}>$ & $/ \mathrm{u} /$ & $\begin{array}{c}\text { /u/ en muchas sílabas cerradas, especialmente ante } \\
\text { nasales no coronales }\end{array}$ \\
\hline$<y>$ & $/ y /$ & /j/ en el diptongo /øj/ (<øy>) \\
\hline$<æ>$ & $/ æ /$ & /e/ en ciertas palabras \\
\hline$<\varnothing>$ & /ø/ & \\
\hline$<a ̊>$ & /o/ & \\
\hline
\end{tabular}

Fuente: elaboración propia.

\section{Diptongos}

De acuerdo con Kristoffersen (2000, p. 19), los diptongos se analizan como secuencias de una vocal corta seguida de una aproximante. Los tres más comunes son $<$ ei $>/ æ j /,<ø y>/ ø j / y<a u>$ $/ æ 0 /$, pero también se encuentran <oi $>$ /oj/, <ui $>/ \mathrm{uj} /$ y $<$ ai $>/ a j /$.

En la tradición lingüística noruega, a diferencia de la española, secuencias de aproximante seguida de vocal nuclear no se consideran diptongos. Por lo tanto, es importante señalar que las aproximantes /j/ y / / también pueden anteceder a cualquiera de las nueve vocales en noruego.

\section{Consonantes}

Los fonemas consonánticos del noruego se presentan en la tabla 3. 
Tabla 3

Fonemas consonánticos del noruego

\begin{tabular}{ccccccc}
\cline { 2 - 5 } & Labial & Dental / alveolar & Retrofleja & Palatal & Velar & Glotal \\
\hline Oclusiva & $\mathrm{p} \mathrm{b}$ & $\mathrm{t} \mathrm{d}$ & $\mathrm{t} \mathrm{d}$ & $\mathrm{k} \mathrm{g}$ & \\
Nasal & $\mathrm{m}$ & $\mathrm{n}$ & $\eta$ & $\mathrm{g}$ & \\
Fricativa & $\mathrm{f}$ & $\mathrm{s}$ & $\mathrm{s}$ & $\mathrm{c}$ & & $\mathrm{h}$ \\
Vibr. simple & & $\mathrm{f}$ & $\mathrm{r}$ & & \\
Aprox. lateral & & $\mathrm{l}$ & $\mathrm{l}$ & & \\
Aproximante & $\mathrm{v}$ & & & $\mathrm{j}$ & \\
\hline
\end{tabular}

Fuente: Kristoffersen (2000, p. 22).

Como se ha mencionado anteriormente, el modelo de Kristoffersen (2000) postula una diferencia entre consonantes moraicas y no moraicas. La moracidad es una propiedad codificada a nivel fonológico. Cuando una consonante moraica se encuentra inmediatamente después de una vocal acentuada, esta vocal siempre se realiza como corta, debido a que la vocal y la consonante proporcionan las dos moras requeridas para una sílaba. Además, si se encuentra otra vocal inmediatamente después de la consonante moraica, ocurre una geminación de la consonante, la cual se vuelve ambisilábica: pasa a formar parte tanto de la coda de la primera de las dos sílabas como del ataque de la segunda. Esto sucede como consecuencia de la interacción entre dos fenómenos: el principio universal de maximización del ataque silábico y el principio particular del noruego de la bimoracidad de las sílabas acentuadas. En cambio, las consonantes no moraicas nunca se geminan y siempre forman parte de la segunda sílaba si se encuentran en posición intervocálica; en estos casos, la vocal precedente se alarga si se le asigna acento léxico. En la mayoría de los casos, las consonantes geminadas, así como las moraicas que se encuentran a final de palabra, se geminan también en la ortografía.

Con respecto al sistema consonántico del español, la diferencia más notable, aparte del fenómeno de la moracidad, es la existencia de una serie de consonantes retroflejas en el noruego. Estas, excepto / $/$ / y la mayoría de las instancias de /s/, derivan históricamente de secuencias de / $/$ / seguida de otra consonante alveolar, lo cual se refleja en la ortografía; por ejemplo, / $t$ / se escribe <rt>. En varios casos se puede afirmar que las retroflejas ya se han fonemizado, pero el proceso sigue siendo productivo, por lo que también surgen alófonos retroflejos cuando coinciden vibrantes simples con otras consonantes alveolares a través de lindes de morfema o de palabra. 
Los fonemas españoles que no tienen correspondencias evidentes en el noruego son la africada palatal sorda $/ \mathrm{t} J$, la fricativa palatal sonora $/ \mathrm{j} /$, la nasal palatal $/ \mathrm{n} /$, la fricativa velar $/ \mathrm{x} /$, la vibrante múltiple alveolar $/ \mathrm{r} / \mathrm{y}$ la aproximante labiovelar /w/, así como la aproximante lateral palatal $/ K /$ de los dialectos lleístas y la fricativa dental sorda $/ \theta /$ de los dialectos de distinción. ${ }^{6}$ Por lo tanto, en el análisis se hará énfasis particular en la adaptación de estos segmentos.

\section{Acento tonal}

A primera vista, la posición del acento léxico principal en el noruego depende de si se trata de una voz patrimonial o de un préstamo. En el primer tipo, el acento prácticamente siempre cae en la primera sílaba cuando la palabra es morfológicamente simple. En los préstamos, por su lado, la posición del acento no es predecible, pero tiende a coincidir con la posición en la lengua fuente. Por lo tanto, tradicionalmente se ha postulado una distinción fonológica entre estos dos grupos de palabras para la asignación de la posición del acento: en las palabras nativas, el acento es predecible con base en la estructura morfológica, mientras que en los préstamos, el acento tendría que estar señalada fonológicamente (cf. Kristoffersen, 2000, p. 148).

No obstante, Rice (1999) propone un modelo, posteriormente desarrollado por Kristoffersen (2000), dentro del marco de la Teoría Métrica que, en lugar de suponer marcación fonológica del acento, puede predecir la posición del acento en la mayoría de las palabras, tanto patrimoniales como préstamos, por medio de un algoritmo.
Casi todas las desviaciones son explicadas mediante una marcación extraordinaria de acento o extraprosodicidad en la sílaba final. El modelo no será descrito con gran detalle en este artículo, pero los resultados del algoritmo se pueden sintetizar de la siguiente manera. Primero, si la palabra es monosilábica, la única sílaba será acentuada. Para las palabras plurisilábicas sin marcación fonológica extraordinaria, el acento cae en la última sílaba si esta es pesada (es decir, bimoraica) antes de la asignación del acento; si no, cae en la penúltima. En cambio, si la última sílaba está marcada como extraprosódica, el acento cae en la penúltima sílaba si esta es pesada y si no, cae en la antepenúltima (en caso de que haya tres sílabas). Asimismo, si la última sílaba está marcada fonológicamente como acentuada, el acento, desde luego, cae en esa sílaba. El hecho de que casi todas las palabras patrimoniales llevan acento en la primera sílaba se debe a que tienden a consistir en una solo sílaba o en una sílaba pesada seguida de una ligera.

Por añadidura, el noruego es una lengua de acento tonal, lo cual quiere decir que el acento primario de una palabra es realizado con una de dos melodías contrastivas. Estas se conocen como tonema 1 y tonema 2 , o bien, acento 1 y acento 2. Existe una gran cantidad de pares mínimos con el acento en la misma sílaba que se distinguen únicamente por el tonema. La realización de los tonemas varía geográficamente. En el dialecto aquí estudiado, de acuerdo con Kristoffersen (2000, p. 236-237), el tonema 1 es realizado mediante un tono bajo al inicio de la sílaba acentuada, el cual asciende a lo largo de esta y la sílaba siguiente, 
por lo que puede representarse como LH ("low-high"). A su vez, el tonema 2 empieza con un tono alto, desciende a lo largo de la sílaba acentuada y vuelve a ascender en la sílaba siguiente, de ahí que sea de tipo HLH ("high-lowhigh”). El tonema 2 requiere de mínimo una sílaba inacentuada o de acento secundario para ser realizado, por lo que no hay contraste tonémico en palabras monosilábicas (Kristoffersen, 2000, p. 233). Convencionalmente, en las transcripciones fonéticas se emplea un número superescrito al inicio de la sílaba acentuada para indicar tanto la posición del acento como el tonema. En cuanto al nivel fonológico, Kristoffersen (2000, p. 253), quien analiza el acento tonal en el marco de la fonología autosegmental, asume que el tonema 1 es el no marcado. De este modo, el acento léxico se realiza como LH por defecto, mientras que las palabras que fonéticamente presentan el tonema 2 llevan un tono alto $(\mathrm{H})$ flotante adicional en la forma subyacente; este se asocia con el patrón LH, lo cual produce la melodía HLH como realización fonética.

\section{Metodología}

En Bokmålsordboka (Universitetet i Bergen y Språkrådet, 2020), el diccionario normativo de la ortografía del noruego bokmål, se realizó una búsqueda avanzada del término "spansk" ('español') dentro de las definiciones. De esta manera, se confeccionó un corpus de todas las palabras señaladas como provenientes del español; 94 en total. Muchas de estas no son voces patrimoniales del español, sino que provienen originalmente de otras lenguas, pero todas están completamente integradas en el sistema fonológico (y el ortográfico) español, por lo que sí se incluyeron en el estudio. El corpus no incluye topónimos.

En ningún diccionario se consigna la ruta tomada por los vocablos desde el español hasta llegar al noruego, pero es probable que una gran parte haya entrado por medio de otra lengua tradicionalmente más influyente en Noruega, sea el inglés, el francés o el alemán. En la mayoría de los préstamos del español, no se detecta ninguna influencia evidente de otra lengua. Sin embargo, para seis de las palabras sí parece ser el caso, por lo que estas se eliminaron de la lista. Se trata de chili (de chile; la última vocal probablemente ha sido influenciada por el inglés, aunque esta forma se aproxima más a la original en náhuatl), kannibal (de caníbal; la geminación ortográfica de la $<$ n $>$ parece haber sucedido por influencia de otra lengua europea), korridor (según Bokmålsordboka, llegó del italiano a través del español, pero mantiene la vocal $i$ del italiano corridore), lasso (de lazo; probablemente con influencia del inglés lasso), sigar (de cigarro; probablemente con influencia del francés cigare o del inglés cigar) y sjokolade (de chocolate; probablemente con influencia del alemán Schokolade).

En segundo lugar, se excluyeron 12 palabras que el investigador no ha escuchado en uso en noruego, por lo cual desconoce cómo sería su pronunciación exacta (aunque se podría predecir con alta probabilidad en la mayoría de los casos): centimo, conga, dublon, gaucho, guano, hacienda, hidalgo, kamarilja, mantilla, paso doble, quipu y zorrilla.

Después de la eliminación de los dos conjuntos de vocablos mencionados, quedó un corpus de 76 palabras. 
Estas se ordenaron en una tabla (véase el anexo) que provee la siguiente información: forma ortográfica en español, forma fonológica en español ${ }^{7}$ (en algunos casos, varias formas fonológicas, para tomar en cuenta la variación dialectal), forma ortográfica en noruego bokmål, forma fonológica en noruego y forma fonética en noruego. En las transcripciones fonológicas del noruego se siguen los mismos principios que en Kristoffersen (2000): no se señala la moracidad consonántica, la extrasilabicidad ni la marcación extraordinaria de acento, aunque sí se sean propiedades fonológicas. Además, se consignan dos transcripciones fonológicas y fonéticas en ciertos casos: la primera es la forma normalmente utilizada por el investigador, y la segunda es una forma alternativa también observada en el mismo dialecto.

En el corpus se puede notar que muchos de los vocablos en noruego mantienen la forma ortográfica del español, mientras que otros han sido más adaptados a la ortografía noruega; inclusive, hay los que presentan dos formas aceptadas. Asimismo, algunos han perdido la vocal final asociada con el género gramatical en español, y algunos han sido prestados en su forma plural con sentido singular (lo que también ha sucedido con varios préstamos del inglés), puesto que la -s no se asocia con plural en noruego. También cabe señalar que, en muchas ocasiones, la semántica de los préstamos ha sido alterada con respecto a su significado en español. Por ejemplo, lugar alude específicamente a un camarote de un barco, eldorado (de El Dorado) a un lugar paradisiaco o de deseo, y maskara (de máscara) a 'rímel'. Sin embargo, por tratarse de un estudio fonológico, no se proporciona el significado de cada palabra.
Finalmente, con base en este corpus, se determinó cómo se ha adaptado cada uno de los fonemas del español al sistema fonológico del noruego y se trató de identificar los patrones en la asignación de acento léxico y tonema. El corpus no ofrece muchos vestigios para determinar el grado de influencia fonológica frente a la fonética en los préstamos, dado que pocos fonemas del español presentan alófonos correspondientes a dos fonemas distintos en el noruego. Por lo tanto, a nivel segmental, el análisis se centró en identificar el grado de influencia ortográfica frente a influencia fonética/fonológica en las adaptaciones.

\section{Análisis}

\section{Vocales}

Ya que el fonema vocálico /a/ suele realizarse como central en español, el fonema noruego fonéticamente más cercano es probablemente /æ/. No obstante, /a/ corresponde invariablemente al fonema noruego /a/ en los préstamos. Un buen ejemplo es armada [ar. ${ }^{1}$ ma: da], que incluye tres instancias diferentes de dicho fonema. Este patrón puede deberse a dos hechos. En primer lugar, el grafema <a> en noruego representa prototípicamente el fonema /a/; solamente en el diptongo <au> (/æo/) representa /æ/. En segundo lugar, /æ/ tiene una distribución limitada, pues prácticamente solo aparece ante $/ \mathrm{f} / \mathrm{/j} /$, $/ \mathrm{l}$ y consonantes retroflejas. De todos modos, queda claro que el factor dominante es la forma ortográfica, puesto que /æj/ sí es una secuencia válida en noruego, pero papaya se pronuncia [pa'1paj.ja], nunca *[pa.1pæj.ja]. 
El fonema español /e/ corresponde al fonema noruego /e/ en todas las palabras del corpus. Además, sus alófonos concuerdan con la distribución descrita anteriormente, como se observa, por ejemplo, en las palabras bolero [bu.1le: $\mathrm{ru}$, embargo [عm.1bar.gu] y tilde [2til. də]. Debido a que el fonema /e/ es representado por el grafema $<\mathrm{e}>$ en ambas lenguas, no se puede determinar si la adaptación en este caso tiene bases fonológicas, fonéticas u ortográficas.

El fonema /i/ del español corresponde a /i/ en noruego en todos los casos (por ejemplo, liga [1li:.ga] y marimba [ma.1rim.ba]), excepto en la palabra mais [1majs] (de maíz), donde representa la aproximante /j/. En noruego, la secuencia <ai> invariablemente representa el diptongo /aj/ (la <i> nunca se tilda en noruego, por lo que este diacrítico se perdió en la importación), y no existen palabras nativas morfológicamente simples con la secuencia bisilábica [ai]. Por lo tanto, esta adaptación podría ser motivada tanto por la fonotaxis noruega como por la ortografía.

A su vez, el fonema español /u/ es sustituido sistemáticamente por / $\mathrm{u} /$ en todo el corpus. Ejemplos son barrakuda [ba.ra.1ku:.da] (de barracuda) y rumba

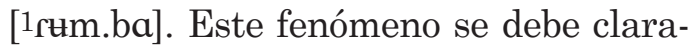
mente a la ortografía, ya que el grafema $<u>$ en noruego representa prototípicamente el fonema $/ \mathrm{u} /$, aunque la [u] noruega, representada prototípicamente por $<_{0}>$, fonéticamente es casi idéntica a la [u] del español. Ahora bien, las secuencias <gu> y <qu>, que en español representan los fonemas /g/ y /k/, respectivamente, se pronuncian sin la vocal también en noruego. En dos casos, la $<\mathrm{u}>$ ni siquiera se escribe: gerilja [ge.1ril.ja] (de guerrilla) y moskito [mu.1ski..tu] (de mosquito). Por su lado, en conquistador [kon.kis.ta.1do:r] y quechua [1ket.su.a] se ha mantenido la forma ortográfica española, pero la $<u>$ tampoco se pronuncia aquí. La ausencia de este fonema vocálico en los préstamos tiene que deberse a influencia fonológica o fonética.

El fonema /o/ del español representa variación en los préstamos en noruego y, como en los casos de las demás vocales, sus adaptaciones se pueden adscribir principalmente a la ortografía. En sílabas abiertas es interpretado como /u/ (con solo una excepción), lo cual corresponde a la pronunciación canónica del grafema $<_{0}>$ en noruego. Ejemplos son bolero [bu.1le:.ru], eldorado [cl. du.1ra:.du] (de El Dorado) y moskito [mu.1ski:.tu]. La única excepción es oregano [o.rə.1ga:.nu] (de orégano), que, por cierto, también es la única palabra del corpus en la que $<0>$ representa una vocal en una sílaba sin consonantes, lo cual podría ser la explicación. En sílabas cerradas, en cambio, hay mucha variación, tanto en las tónicas como en las átonas. En voces como bongo [1bon. gu] y conquistador [koy.kis.ta.1do:r], es más general la pronunciación con /o/, especialmente en la última sílaba de conquistador. ${ }^{8}$ En contraste, kondor [kun.1du:r] (de cóndor) y sombrero [sum.1bre:.ru] son pronunciadas con [u] por el investigador. Esta irregularidad es, sin duda, un resultado de que <0> en la ortografía noruega puede representar tanto /o/ como /u/ en sílabas cerradas, por lo que el grado de influencia fonológica/fonética ${ }^{9}$ de la lengua fuente determina la vocal empleada.

\section{Diptongos}

Los diptongos crecientes españoles no son interpretados como diptongos en noruego. Esto queda claro en palabras 
como donjuan [don.su.1an] (de don Juan) y siesta [si.1Es.ta]. En todos los casos, las dos vocales gráficas se interpretan como dos vocales silábicas en noruego, por lo que se incrementa el número de sílabas. La secuencia [wa] es inexistente en noruego, mientras que [je] sí es permitida, pero no después de /s/; en consecuencia, la desdiptongación puede suceder por razones tanto ortográficas como fonotácticas.

\section{Consonantes}

Los siguientes fonemas consonánticos del español corresponden a fonemas noruegos con realizaciones muy similares, y que suelen ser representados por los mismos símbolos: /p/, /t/, /d/, /k/, /g/, /f/, /s/, /m/, /n/, /s/ y /l/. Por supuesto, las realizaciones fonéticas exactas no son las mismas en ambos idiomas, pero no se ahondará en tales detalles en este artículo. Asimismo, la debilitación experimentada por las oclusivas sonoras en ciertos entornos en el español no ocurre en noruego, por lo que estos fonemas siempre se realizan como oclusivas. No es posible determinar si las adaptaciones de estas consonantes se deben a razones fonológicas, fonéticas u ortográficas.

Con respecto a /b/, este fonema se representa ortográficamente en español de dos maneras: $<\mathrm{b}>\mathrm{y}<\mathrm{v}>$. En noruego, $<\mathrm{v}>$ siempre representa la aproximante /o/, razón por la cual la $<\mathrm{v}>$ en las únicas palabras del corpus que contienen dicho grafema, avocado [a.vu.1ka:.du] y centavo [sen.1ta:.vu], ha sido interpretada como/v/ en los préstamos.

También cabe mencionar que, en varias palabras del corpus, el fonema /n/ es realizado como [n] en español, debido a que se encuentra ante una consonante velar. Esta misma asimilación tiene lugar en noruego, por ejemplo en flamenco [fla.1men.ku]. Por tanto, en estos casos, /n/ se neutraliza con /y/.

En lo que concierne al fonema $/ \theta /$, presente en gran parte de España, este siempre corresponde a /s/ en noruego, al igual que en las variedades seseantes del español. Dos ejemplos son mestis [me.1sti:s] (de mestizo) y sigarillo [si. ga.1ril.lu] (de cigarrillo). No se puede determinar si esto se debe a influencia fonética/fonológica de dialectos españoles seseantes, si se trata de una aproximación al fonema noruego más similar o si es otro caso más de influencia ortográfica, pues los nombres de las letras $<c>$ y $<$ z $>$ (no utilizadas en vocablos patrimoniales) en noruego son [1 $\mathrm{se}:]$ y [1sct] (ambos con el fonema /s/), respectivamente.

Como ya se ha apreciado, la variedad noruega tratada aquí no posee ninguna africada. En consecuencia, la adaptación fonológica del fonema /t Jt merece atención particular. Se observan dos estrategias: la sustitución por una secuencia de dos fonemas, /ts/, y la simplificación articulatoria a favor de la fricativa retrofleja /s/. La primera se fundamenta en mayor similitud fonética, mientras que la segunda consiste en una sustitución de un fonema por otro. Sin embargo, la última opción no necesariamente implica una interpretación fonológica de /t Jt como un solo fonema en español, sino que se puede explicar por la fonotaxis del noruego, la cual, tradicionalmente, no permite la secuencia /ts/ en una misma sílaba. De todos modos, esta sí se ha vuelto más común con la entrada de varios préstamos que contienen africadas en la lengua fuente, sobre todo del inglés, por lo que esta restricción fonotáctica podría encontrarse en proceso de 
modificación. ${ }^{10}$ En el corpus, cuando se emplea la opción de /tș/, en primer lugar, la /t/ se asimila al punto retroflejo de la /s/. Además, en posición intervocálica, las dos consonantes son divididas por el linde silábico, lo cual deja claro que no se trata de un fonema africado en noruego. Esta misma secuencia se puede encontrar a través de lindes de palabra en voces patrimoniales, así como en la onomatopeya que representa un estornudo: atsjo [at.1su:]. Con respecto a las palabras del corpus, el investigador utiliza solo la fricativa en las palabras chinchilla [sin. ${ }^{1}$ sil. la] y machete [ma.2se:.tə], pero estas también se escuchan frecuentemente con la secuencia /ts/. Por el contrario, las palabras macho [1mat.su] y quechua [1ket.su.a], donde el fonema español / tJt se halla en posición intervocálica, no suenan aceptables sin el segmento oclusivo. Finalmente, el investigador pronuncia poncho [1pon.tsu] con /ts/, posiblemente por su posición posnasal. De todos modos, en suma, la parte oclusiva /tJ/ parece mantenerse en posición intervocálica, donde no irrespeta la fonotaxis noruega, mientras que es más frecuentemente elidida en otras posiciones.

Otro fenómeno que merece mencionarse es que /sk/ en moskito [mu.1ski:. tu] (de mosquito) no sea sustituida por /S/ a pesar de que la secuencia de grafemas $<$ sk $>$ seguida de una vocal anterior normalmente represente dicho fonema en noruego. De hecho, en noruego tradicionalmente no se permite la secuencia/ski/ en un mismo morfema, ya que esta, históricamente, es el origen de la secuencia/si/. Empero, también en este caso, se nota que la fonotaxis se encuentra en proceso de cambio debido a préstamos de varios idiomas en los que sí existe dicha combinación de fonemas.
Como se ha apreciado, el noruego no tiene contraste fonológico entre vibrante simple y múltiple: solo existe la vibrante simple / $/$ (que puede geminarse en caso de ser moraica). En las tres palabras del corpus que incluyen una vibrante múltiple en español, esta es sustituida por la vibrante simple en noruego (a pesar de que se mantenga ortográficamente la doble $<\mathrm{r}>$ en barrakuda): barrakuda [ba.ra. ${ }^{1 \mathrm{ku}}$ : da], gerilja [ge.1ril.ja] (de guerrilla) y sigarillo [si.ga.1ril.lu].

En la palabra tortilla, la vibrante simple ocurre ante una consonante alveolar, secuencia que en noruego produce una consonante retrofleja. Este préstamo, efectivamente, presenta la pronunciación [tu.1tij.ja] o [tu.1til.la]. El uso de la retrofleja en este vocablo debe ser el resultado de una interpretación de la secuencia gráfica $<$ rt $>$ como representante del fonema retroflejo $/ \mathrm{t}$.

Los fonemas noruegos articulatoriamente más cercanos a la $/ \mathrm{x} / \mathrm{del}$ español son la fricativa retrofleja /s / y la fricativa palatal/ç/. En donjuan [don.su.1an], la elección es la retrofleja /s/. Pareciera tratarse de un caso de influencia fonológica/fonética del español, pero también podría ser una analogía con préstamos del francés, en los que la $<j>$ (que normalmente representa /3/ en el francés) se ha adaptado como /s/ debido a la inexistencia de fricativas sonoras en el noruego. Por otro lado, en las otras dos palabras del corpus que contienen /x/ en español, el correspondiente en noruego es la aproximante palatal /j/: jade [2ja:də] y junta [1jun.ta]. Esto sucede, evidentemente, por influencia ortográfica, ya que la letra /j/ representa el fonema /j/ en noruego.

El análisis de los fonemas españoles /j/ y /K/ es más complejo, puesto que en 
la mayoría de las variedades del español han confluido en el fonema /j/. En lo que concierne a papaya, con el fonema /j/ en todas las variedades del español, la correspondencia en noruego es la aproximante palatal /j/: papaya [pa.1paj.ja]. Asimismo, como se puede apreciar por la geminación, esta aproximante se interpreta como moraica, al igual que cuando forma parte de diptongos en voces patrimoniales noruegas. Es difícil determinar si esta correspondencia se debe a la similitud fonética con el diptongo noruego <ai> /aj/ o a la coincidencia con la $<y>$ en el diptongo <øy> /øj/ (como en <øуa $>$ [1øj.ja] 'la isla').

En lo que atañe al dígrafo $<11>$ del español, este representa el fonema $/ K /$ en ciertas variedades del español y /j/ en otras. Curiosamente, en muchos cursos de español en Noruega, la pronunciación es descrita explícitamente como [lj]; ${ }^{11}$ es decir, una aproximación a la del fonema $/ K /$, a pesar de que la mayoría de los dialectos españoles hoy en día, inclusive en España, sean yeístas. Esta puede ser la causa del uso de la secuencia de /lj/ en gerilja [ge.1ril.ja]. Además, cabe señalar que /lj/ también se encuentra en posición intervocálica en varias palabras patrimoniales noruegas. Por otro lado, una aproximación al habla yeísta, mediante /j/, parece ser lo más común en paella [pa.1عj.ja] y tortilla [tu.1tij.ja], aunque las formas [pa.1عl.ja] y [tu.1til.la] tampoco son raras. En cambio, en chinchilla [sin.1sil. la], sigarillo [si.ga.1ril.lu] y la variante tortilla [tu.1til.la], donde el fonema en cuestión ha sido interpretado como una /l/ moraica, la explicación es indudablemente ortográfica. Por último, la palabra española llama lleva el fonema palatal en posición inicial, donde en noruego no puede aparecer la secuencia /lj/ ni una consonante geminada. El resultado es el fonema /1/: lama [1la:.ma].

En la única palabra del corpus que contiene la nasal palatal /n/ se observa una clara aproximación fonológica/ fonética en la adaptación al noruego: castañeta se ha tomado prestada como kastanjett [kas.tan.1jet], con una sustitución de /n/ por la secuencia /nj/. El mismo fenómeno se observa en topónimos como Spania/spanja/ [1spa:n.ja] (de España).

El último tema por tratar en este subapartado es la moracidad de las consonantes, la cual es reflejada superficialmente por medio de la geminación de una consonante en posición posvocálica en sílaba acentuada, o bien, la realización breve de una vocal en sílaba cerrada acentuada cuando no le sigue otra sílaba con consonante inicial. En posición interior de palabra, tres consonantes se manifiestan como moraicas en el corpus. En primer lugar, cuando la $/ K /$ del español se interpreta como /l/ en noruego, esta siempre es moraica, claramente por influencia de la grafía <ll>: chinchilla [sin. ${ }^{1}$ sil.la], sigarillo [si.ga.1ril.lu] y tortilla [tu.1til. la]. En segundo lugar, la aproximante /j/ siempre se manifiesta como moraica cuando se halla en posición intervocálica: paella [pa.1عj.ja], papaya [pa.paj. ja] y tortilla: [tu.11ij.ja]. Este comportamiento se observa con el mismo fonema en todos los préstamos. En tercer lugar, el fonema /k/ también se interpreta como moraica en varios de los préstamos: alpakka [al.1pak.ka] (de alpaca), coca [1kuk.ka] y kokos [1kuk. kus] (de cocos). Además, una pronunciación alternativa común de kakao es [1kak.ka.u], en la que se observa que la $/ \mathrm{k} /$ se gemina cuando el acento se pasa 
a la primera sílaba. Solo en un vocablo -taco [1ta:ku]- la /k/ no es moraica en este entorno. Los patrones observados son relevantes para la comprensión general de las consonantes moraicas y merecen mayor atención en estudios de adaptación de préstamos de otras lenguas al noruego.

En cuanto a consonantes moraicas en posición final de palabra, se observan tres: kastanjett [kas.tan.1jet], mulatt [mu.1lat] (de mulato) y donjuan [don. su.1an]. Las dos primeras son los únicos dos casos de una consonante oclusiva oral en posición final de palabra en el corpus, lo cual, junto con los datos de la /k/ ya tratados, constituye una indicación de que las oclusivas, especialmente las sordas, son más propensas a ser moraicas que otras clases de consonantes (excepto las aproximantes).

\section{Acento tonal}

La posición del acento léxico en los préstamos es la misma que en las palabras españolas en la mayoría de los casos. Las únicas excepciones son kano [1ka:.nu] (de canoa), kolibri [ku.1li:. bri] (de colibri; pero también se observa la forma [ku.li.1bri:]), kondor [kun.1duir], mascara [ma.1skai.ra] y oregano [o.rə.1ga:.nu] (además de mais [1majs], reinterpretada como monosilábica, como señalado anteriormente). Sin embargo, todos estos casos, aparte de kondor, cumplen con la regla básica de asignación de acento de Kristoffersen (2000), lo que explicaría por qué se desvían de su pronunciación original. En el caso particular de kondor, la regla básica predice que se acentúe en la primera sílaba, puesto que la coda de la última sílaba es constituida por una consonante no moraica. Esto quiere decir que la última sílaba lleva marcación extraordinaria de acento, lo cual podría deberse a una analogía con el préstamo kontor [kun.1tu:r] 'oficina' o con otros préstamos terminados en <dor> (conquistador, matador, etc.).

Muchas más de las palabras llevan marcación excepcional de extrasilabicidad o de acento, pero en todos estos casos, su finalidad parece ser mantener la posición del acento de la palabra española. Más frecuentemente, se trata de marcación de acento en la última sílaba cuando esta contiene una consonante no moraica en la coda, lo que siempre resulta en una vocal larga; por ejemplo, banan [ba.1na:n] (de banana/banano), conquistador [kon.kis.ta.1do:r], lugar [lu.1ga:r] y mestis [me.1sti:s]. Asimismo, se encuentran manifestaciones de sílaba final extraprosódica en las palabras indigo [1in.di.gu] (de índigo), nutria [1nu:.tri.a] y quechua [1ket.su.a].

En resumen, en la mayoría de las ocasiones, la regla básica de asignación de acento del noruego acentúa la misma sílaba que es la acentuada en español. En caso contrario, las marcaciones extraordinarias en los préstamos sirven para mantener la posición del acento de la lengua fuente (con la excepción de kondor). Debido a que muchas de las palabras no llevan acento gráfico en español, además del hecho de que dicha marca diacrítica es poco común en noruego, se debe concluir que la posición del acento en los préstamos presenta una influencia fonológica/fonética notable. Por otro lado, la ausencia de marcación extraordinaria en algunos otros préstamos, que, en consecuencia, presentan menor grado de influencia fonológica/fonética, puede causar que el acento caiga en una sílaba diferente en noruego que en español. 
En lo que concierne a los tonemas, casi todas las palabras del corpus presentan el tonema 1. Esto sugiere que dicho tonema es el que se asigna por defecto a los préstamos, lo cual es lógico si este se considera el tonema no marcado. Las cuatro excepciones, con el tonema 2, son las siguientes: jade [2ja:də], tilde [2til.də], machete [ma.2se:.tə] y sangria [say.2gri:a]. Las primeras tres conforman claramente un grupo distintivo: son las únicas palabras del corpus que terminan en /e/. Efectivamente, Kristoffersen (2000, p. 256) nota que el patrón productivo para palabras bisilábicas acabadas en esta vocal (lo cual corresponde a jade y tilde) es el tonema 2. Respecto a palabras de más de dos sílabas, el autor también señala que las que tienen una /e/ final usualmente son asignadas el tonema 2; esto sucede con machete. La presencia del tonema 2 en sangria es más difícil de explicar, puesto que Kristoffersen (2000, p. 257) afirma conocer solo una palabra morfológicamente simple acabada en una vocal diferente de /e/ que tenga el tonema 2. Una posible explicación podría ser que el tonema 2 en sangria se deba a una analogía con sustantivos femeninos en su forma singular definido, marcada por medio del sufijo /-a/, ya que estas sí llevan el tonema 2. ${ }^{12}$

\section{Conclusiones}

Con respecto a los fonemas vocálicos, se ha observado una fuerte influencia de la ortografía; de hecho, todas las adaptaciones, con la excepción de ciertas instancias del fonema español /o/ y del grafema $<\mathrm{u}>$, podrían basarse únicamente en la asociación de cada grafema con su fonema canónico en noruego. Por añadidura, los diptongos del español se interpretan como dos vocales silábicas en noruego, lo cual, probablemente, también se debe a las formas ortográficas. Las correspondencias entre los fonemas vocálicos del español con los del noruego se resumen en la tabla 4 .

\section{Tabla 4}

Correspondencias de los fonemas vocálicos españoles en noruego

\begin{tabular}{cc}
\hline Español & Noruego \\
\hline$/ \mathrm{i} /$ & $/ \mathrm{i} /, / \mathrm{j} /$ \\
$/ \mathrm{e} /$ & $/ \mathrm{e} /$ \\
$/ \mathrm{a} /$ & $/ \mathrm{a} /$ \\
$/ \mathrm{o} /$ & $/ \mathrm{u} /, / \mathrm{o} /$ \\
$\mathrm{lu} /$ & $/ \mathrm{u} /$ \\
\hline
\end{tabular}

Fuente: elaboración propia.

En cuanto a los fonemas consonánticos, también hay un alto grado de influencia ortográfica, pero también en estos casos es difícil distinguirla de influencia fonológica/fonética. Sin embargo, también se encuentran casos en los que la ortografía se puede descartar como factor. Esto sucede, en primer lugar, con el fonema español /tJt, que se adapta como /ts/ o /s/ en noruego; en este caso, una influencia ortográfica no sería posible, puesto que la grafía $<$ ch $>$ tradicionalmente no existe en noruego. Asimismo, el empleo de /ts/ es más común en posición intervocálica, donde ambos fonemas pueden separarse en dos sílabas distintas. En segundo lugar, hay una clara influencia no 
ortográfica en algunos préstamos que es /j/ en noruego, por influencia de la originalmente en español contenían ortografía. Las correspondencias entre el fonema $/ K /$, adaptado como $/ \mathrm{lj} /$, así los fonemas consonánticos del español como en la adaptación de /n/ como /nj/. con los del noruego se resumen en la Finalmente, la sustitución de /x/ por /s/ tabla 4 (se ponen entre paréntesis los se basa posiblemente en similitud fo- fonemas que no existen en todas las nológica/fonética, pero en otros casos, variedades del español y las corresponla correspondencia de la /x/ española dencias de estos en noruego):

\section{Tabla 5}

\section{Correspondencias de los fonemas consonánticos españoles en noruego}

\begin{tabular}{cc}
\hline Español & Noruego \\
\hline$/ \mathrm{p} /$ & $/ \mathrm{p} /$ \\
$/ \mathrm{b} /$ & $/ \mathrm{b} /$ \\
$/ \mathrm{t} /$ & $/ \mathrm{t} /$ \\
$/ \mathrm{d} /$ & $/ \mathrm{d} /$ \\
$/ \mathrm{k} /$ & $/ \mathrm{k} /$ \\
$/ \mathrm{g} /$ & $/ \mathrm{g} /$ \\
$/ \mathrm{f} /$ & $/ \mathrm{f} /$ \\
$(/ \theta /)$ & $(/ \mathrm{s} /)$ \\
$/ \mathrm{s} /$ & $/ \mathrm{s} /$ \\
$/ \mathrm{j} /$ & $/ \mathrm{j} /(/ \mathrm{lj} /, / \mathrm{l} /)$ \\
$/ \mathrm{x} /$ & $/ \mathrm{j} /, / \mathrm{s} /$ \\
$/ \mathrm{t} J /$ & $/ \mathrm{ts} /, / \mathrm{s} /$ \\
$/ \mathrm{m} /$ & $/ \mathrm{m} /$ \\
$/ \mathrm{n} /$ & $/ \mathrm{n} /$ \\
$/ \mathrm{n} /$ & $/ \mathrm{hj} /$ \\
$/ \mathrm{f} /$ & $/ \mathrm{f} /$ \\
$/ \mathrm{r} /$ & $(/ \mathrm{j} / / / \mathrm{l} /)$ \\
\hline$/ \mathrm{l} /)$ & \\
\hline & 1 \\
\hline &
\end{tabular}

Fuente: elaboración propia. 
Cabe añadir que es llamativo que algunas de las consonantes sean interpretadas como moraicas pese a que ese rasgo no exista en español. La moracidad consonántica en el noruego es definitivamente un fenómeno que merece estudiarse con mayor detalle.

En la mayoría de las ocasiones, la regla básica de asignación del acento léxico del noruego coloca el acento en la misma sílaba que en la palabra española. Cuando esto no es el caso, muchas veces se emplean los recursos de marcación extraordinaria de acento o extrasilabicidad para que se mantenga la posición del acento de la lengua fuente. Por lo tanto, el mayor grado de influencia fonológica/fonética en la adaptación de los préstamos del español parece encontrarse justamente en la posición del acento.

En lo que atañe a los tonemas, a casi todos los vocablos del corpus se les asigna el tonema 1; solo cuatro palabras presentan el tonema 2. Esto confirma que el tonema 1 es el no marcado, mientras que las instancias del tonema 2 parecen darse analógicamente.

En síntesis, se ha mostrado una fuerte influencia de la ortografía en los préstamos del español en el noruego. A nivel segmental, la adaptación fonológica se desvía de lo sugerido por la ortografía solo en ciertos casos; esto es casi siempre cuando la grafía del español no existe en el noruego o cuando un mismo grafema representa diversos fonemas. La única adaptación que incumple con la fonotaxis tradicional es la secuencia/ts/ (de /t5/) en ataque silábico, pero en estos casos tiende a emplearse solo la fricativa /s/. A nivel suprasegmental, se ha mostrado una influencia fonológica/fonética notable en cuanto a la posición original del acento léxico en español, pero también existen desviaciones.

La conclusión quizá más importante de esta investigación es el hecho de que la forma ortográfica parece ser mucho más influyente de lo que se ha estipulado en estudios anteriores de la adaptación fonológica de préstamos. Por lo tanto, en futuros estudios, sobre todo de préstamos entre lenguas con poco contacto y pocos hablantes bilingües en común, debe prestarse mayor atención a este factor.

\section{Notas}

1. El noruego es una lengua germánica hablada por casi 5 millones de personas, principalmente en Noruega, donde es la única lengua administrativa a nivel nacional.

2. Se reconocen dos ortografías oficiales para el noruego, el bokmål y el nynorsk, ambas reguladas por el mismo ente, Språkrådet.

3. Cita original: "Lånord fra andre språk enn engelsk uttales med lyder som allerede finnes i norsk fonologi".

4. Por ejemplo, la letra $\langle a>$ es denominada [1 ${ }_{\mathrm{a}}$ :], la $<\mathrm{e}>$ es denominada [1e:], etcétera.

5. Kristoffersen (2000) incluye también el fonema aproximante /w/, que aparece únicamente en el diptongo /æw/. Sin embargo, el presente autor pronuncia dicho diptongo como /æv/, por lo que está claro que /w/ se ha fusionado con la aproximante labiodental /o/ en la variedad bajo estudio.

6. Todos estos fonemas son representados por diversos otros símbolos en diferentes publicaciones.

7. En la transcripción fonológica de las palabras españolas, las aproximantes /j/ y 
/w/ se consideran fonemas distintos de las vocales /i/ y /u/ en todos los casos.

8. Aunque, posiblemente, se podrían dar pronunciaciones de las primeras sílabas de estas palabras con [u].

9. Pero no el grado de conocimiento del español de cada hablante en particular, puesto que el investigador es hablante fluido del español, y aun así pronuncia algunas de estas palabras con [u].

10. En algunos casos, estas palabras se escriben con <tsj>, como en Tsjekkia 'Chequia'; aun así, el investigador pronuncia dicho vocablo con/š/inicial: [1 ${ }_{\text {sek.ki.a]. }}$

11. Un ejemplo es en una página sobre fonética española en la Universidad de Oslo (Institutt for litteratur, områdestudier og europeiske språk, s.f.).

12. En su forma base, llevan el sufijo /-e/ como marca de género femenino singular indefinido. Cuando este es sustituido por /-a/ para marcar el definido, el tonema asociado con la raíz se mantiene.

\section{Bibliografía}

Andersen, P. H. (2007). Oh bloody hell sir æ åsså tar av mæ kappa. En undersøkelse av engelske innslag i rollespill [Tesis de maestría, Universidad de Tromsø].

Brandsegg, T. (2001). Kolles pute'n herre together da? - om engelske innslag i talespråket til trønderske ungdommer [Tesis de maestría, Universidad Noruega de Ciencia y Tecnología].

Calabrese, A. y Wetzels, W. L. (2009). Loan phonology. Issues and controversies. En Calabrese, A. y Wetzels, W. L. (Eds.), Loan phonology (pp. 1-10). John Benjamins.

Chang, C. (2008). Phonetics vs. Phonology in Loanword Adaptation:
Revisiting the Role of the Bilingual. UC Berkeley Phonology Lab Annual Report (2008).

Endresen, R. T. (1991). Fonetikk og fonologi. Ei elementær innføring ( $2^{\mathrm{a}}$ ed.). Universitetsforlaget.

Feke, M. S. (2003). Adaptaciones fonéticas quechuas de préstamos léxicos españoles. Revista Andina, 37(2), 237-247.

Foss, E. S. (2019). Spansk mest populært. https://www.ssb.no/utdanning/artikler-og-publikasjoner/ spansk-mest-populaert

Hualde, J. I. (2009). Phonologically unmotivated changes in language contact: Spanish borrowings in Basque. Folia Linguistica, 27(1-2), 1-26. https://doi. org/10.1515/flin.1993.27.1-2.1

Institutt for litteratur, områdestudier og europeiske språk, Det humanistiske fakultet, Universitetet i Oslo. (s.f.). Fonética | Fonetikk. https:// www.hf.uio.no/ilos/tjenester/ kunnskap/sprak/nettsprak/spansk/ portal/spa1101/fonetikk/

Kristoffersen, G. (2000). The Phonology of Norwegian. Oxford University Press.

LaCharité, D. y Paradis, C. (2005). Category preservation and proximity versus phonetic approximation in loanword adaptation. Linguistic Inquiry, 36(2), 223-258. https://doi. org/10.1162/0024389053710666

Lea, A. H. (2009). Lånord i norsk talespråk [Tesis de maestría, Universidad de Oslo].

Peperkamp, S. (2005). A psycholinguistic theory on loanword adaptations. En Ettlinger, M., Fleischer, N. y Park-Doob, M. (Eds.), Proceedings of the 30th Annual Meeting of the Berkeley Linguistics Society (pp. 341-352). Berkeley Linguistic Society. 
Pinta, J. D. (2013). Lexical strata in loanword phonology: Spanish loans in Guarani [Tesis de maestría, University of North Carolina at Chapel Hill].

Rice, C. (1999). Norwegian. En van der Hulst, H. (Ed.), Word prosidic systems in the languages of Europe (pp. 545-553). Mouton de Gruyter.

San Giacomo, M. y Peperkamp, S. (2008). Presencia del español en náhuatl: estudio sociolingüístico de la adaptación de préstamos. En Westmoreland, M. y Thomas, J. A. (Eds.), Selected Proceedings of the 4th Workshop on Spanish Sociolinguistics (pp. 149-156).

Sayahi, L. (2005). Phonological adaptation of Spanish loanwords in
Northern Moroccan Arabic. U. Penn Working Papers in Linguistics, 11(1), 253-261.

Silverman, D. (1992). Multiple scansions in loanword phonology: evidence from Cantonese. Lingua 116(7), 1046-1078.

Torp, A. y Vikør, L. S. (2000). Hovuddrag i norsk språkhistorie (2 ${ }^{\mathrm{a}}$ ed.). Gyldendal Akademisk.

Universitetet i Bergen y Språkrådet. (2020). Bokmålsordboka. ordbok. uib.no

Vendelin, I. y Peperkamp, S. (2006). The influence of orthography on loanword adaptations. Lingua, 116, 996-1007. https://doi.org/10.1016/j. lingua.2005.07.005 


\section{El corpus}

\begin{tabular}{|c|c|c|c|c|}
\hline $\begin{array}{c}\text { Forma } \\
\text { ortográfica en } \\
\text { español }\end{array}$ & $\begin{array}{c}\text { Forma } \\
\text { fonológica en } \\
\text { español }\end{array}$ & $\begin{array}{c}\text { Forma } \\
\text { ortográfica en } \\
\text { noruego }\end{array}$ & $\begin{array}{c}\text { Forma } \\
\text { fonológica en } \\
\text { noruego }\end{array}$ & $\begin{array}{c}\text { Forma } \\
\text { fonética en } \\
\text { noruego }\end{array}$ \\
\hline albino & /albino/ & albino & /albinu/ & [al.1bi:.nu] \\
\hline alpaca & /alpaka/ & alpakka & /alpaka/ & [al.1pak.ka] \\
\hline armada & /armada/ & armada & /armada/ & [ar.1ma:.da] \\
\hline avocado & /abokado/ & avokado & /avukadu/ & [a.vu. $\left.{ }^{1} \mathrm{ka}: . \mathrm{du}\right]$ \\
\hline bacalao & /bakalao/ & $\begin{array}{l}\text { bacalao } \\
\text { bakalao }\end{array}$ & /bakalau/ & [ba.ka.1la:.u] \\
\hline balsa & /balsa/ & balsa & /balsa/ & [1bal.sa] \\
\hline $\begin{array}{l}\text { banana } \\
\text { banano }\end{array}$ & $\begin{array}{l}\text { /banana/ } \\
\text { /banano/ }\end{array}$ & banan & /banan/ & [ba.1na:n] \\
\hline barracuda & /barakuda/ & barrakuda & /barakuda/ & [ba.ra.1ku:.da] \\
\hline bodega & /bodega/ & bodega & /budega/ & [bu.1de:.ga] \\
\hline bolero & /bolero/ & bolero & /buleru/ & [bu.1le:.ru] \\
\hline bongo & /bongo/ & bongo & /bongu/ & [1boy.gu] \\
\hline cacao & /kakao/ & kakao & /kakau/ & $\begin{array}{l}{\left[\mathrm{ka} \cdot{ }^{1} \mathrm{ka}: . \mathrm{u}\right]} \\
{\left[{ }^{\mathrm{kak}} \cdot \mathrm{ka} . \mathrm{u}\right]}\end{array}$ \\
\hline canasta & /kanasta/ & canasta & /kanasta/ & [ka. ${ }^{1}$ nas.ta] \\
\hline canoa & /kanoa/ & kano & /kanu/ & [1ka:.nu] \\
\hline casco & /kasko/ & kasko & /kasku/ & [1kas.ku] \\
\hline castañeta & /kastaneta/ & kastanjett & /kastanjet/ & [kas.tan. $1 \mathrm{j} \varepsilon \mathrm{t}]$ \\
\hline caymán & /kajman/ & kaiman & /kajman/ & [kaj. ${ }^{1 m a: n]}$ \\
\hline centavo & $\begin{array}{l}\text { /Oentabo/ } \\
\text { /sentabo/ }\end{array}$ & centavo & /sentavu/ & [sen.1ta:.vu] \\
\hline chinchilla & 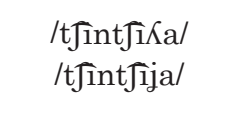 & chinchilla & $\begin{array}{l}\text { /sinsila/ } \\
\text { /tsintşila/ }\end{array}$ & $\begin{array}{c}\text { [sin. }{ }^{1} \text { sil.la] } \\
\left.\text { [tsin. }{ }^{1}{ }^{t} \operatorname{sil} . l a\right]\end{array}$ \\
\hline cigarrillo & $\begin{array}{l}\text { /Oigariאo/ } \\
\text { /Oigarijo/ } \\
\text { /sigariאo/ } \\
\text { /sigarijo/ }\end{array}$ & sigarillo & /sigarilu/ & [si.ga. $1_{\text {ril.lu] }}$ \\
\hline
\end{tabular}




\begin{tabular}{|c|c|c|c|c|}
\hline coca & /koka/ & koka & /kuka/ & [1kuk.ka] \\
\hline $\operatorname{coco}(\mathrm{s})$ & /koko(s)/ & kokos & /kukus/ & [1kuk.kus] \\
\hline colibrí & /kolibri/ & kolibri & /kulibri/ & $\begin{array}{l}\text { [ku.1li:.bri] } \\
\text { [ku.li.1bri:] }\end{array}$ \\
\hline cóndor & /kondor/ & kondor & /kundur/ & [kun. ${ }^{1 \mathrm{du}: \mathrm{r}]}$ \\
\hline conquistador & /konkistador/ & conquistador & /konkistador/ & [koy.kis.ta. ${ }^{1}$ do:r] \\
\hline desperado & /desperado/ & desperado & /desperadu/ & [des.pə.1 $\left.1_{\mathrm{ra}} . \mathrm{du}\right]$ \\
\hline don Juan & /donxwan/ & $\begin{array}{l}\text { donjuan } \\
\text { don juan }\end{array}$ & /donşun/ & [don.st.1 $1 \mathrm{an}$ ] \\
\hline El Dorado & /eldorado/ & eldorado & /elduradu/ & [el.du. $1_{\text {ra:.du }}$ \\
\hline embargo & /embargo/ & embargo & /embargu/ & [عm.1bar.gu] \\
\hline fandango & /fandango/ & fandango & /fandangu/ & [fan. 1 day.gu] \\
\hline flamenco & /flamenko/ & flamenco & /flamenku/ & [fla. ${ }^{1 m \varepsilon y . k u] ~}$ \\
\hline gringo & /gringo/ & gringo & /gringu/ & [1grin.gu] \\
\hline guerrilla & $\begin{array}{l}\text { /geriKa/ } \\
\text { /gerija/ }\end{array}$ & gerilja & /gerilja/ & [ge. $1_{\text {ril.ja] }}$ \\
\hline índigo & /indigo/ & indigo & /indigu/ & [1in.di.gu] \\
\hline jade & /xade/ & jade & /ja:de/ & [2ja:də] \\
\hline junta & /xunta/ & junta & /junta/ & {$[1 \mathrm{jun} . t a]$} \\
\hline liga & /liga/ & liga & /liga/ & [1li:.ga] \\
\hline llama & $\begin{array}{l}\text { /Kama/ } \\
\text { /jama/ }\end{array}$ & lama & /lama/ & [1la:.ma] \\
\hline lugar & /lugar/ & lugar & /lugar/ & [lu.1ga:r] \\
\hline machete & /matJete/ & machete & $\begin{array}{l}\text { /masete/ } \\
\text { /matsete/ }\end{array}$ & $\begin{array}{l}{\left[\mathrm{ma} .{ }^{2} \mathrm{se}: . \mathrm{t} ə\right]} \\
{\left[\mathrm{mat} .{ }^{2} \mathrm{se}: . \mathrm{to}\right]}\end{array}$ \\
\hline macho & /mat5o/ & macho & /matşu/ & [1mat.su] \\
\hline maíz & $\begin{array}{l}\text { /mai } / \text { / } \\
\text { /mais/ }\end{array}$ & mais & /majs/ & [1majs] \\
\hline marimba & /marimba/ & marimba & /marimba/ & [ma. $1_{\text {rim.ba] }}$ \\
\hline marina & /marina/ & marina & /marina/ & [ma.11i:.na] \\
\hline máscara & /maskara/ & mascara & /maskara/ & [ma.1ska:.ra] \\
\hline matador & /matador/ & matador & /matador/ & [ma.ta. ${ }^{1}$ do:r] \\
\hline
\end{tabular}




\begin{tabular}{|c|c|c|c|c|}
\hline merino & /merino/ & merino & /merinu/ & [me.1 1ri:.nu] \\
\hline mesana & /mesana/ & mesan & /mesan/ & {$\left[\mathrm{me} .1_{\mathrm{sa}} \mathrm{n}\right]$} \\
\hline mestizo & 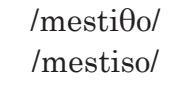 & mestis & /mestis/ & {$\left[\mathrm{m} \varepsilon .1_{\text {sti:s }}\right]$} \\
\hline mosquito & /moskito/ & moskito & /muskitu/ & [mu.1ski:.tu] \\
\hline mulato & /mulato/ & mulatt & /mulat/ & [mu.1lat] \\
\hline nutria & /nutrja/ & nutria & /nutria/ & [1nu:.tri.a] \\
\hline orégano & /oregano/ & oregano & /oreganu/ & [o.rə.1ga:.nu] \\
\hline paella & $\begin{array}{l}\text { /paeKa/ } \\
\text { /paeja/ }\end{array}$ & paella & $\begin{array}{l}\text { /paeja/ } \\
\text { /paelja/ }\end{array}$ & $\begin{array}{l}\left.\text { [pa. }{ }^{1} \varepsilon j . j a\right] \\
{\left[p a .{ }^{1} \varepsilon l . j a\right]}\end{array}$ \\
\hline pampa(s) & /pampa(s)/ & pampas & /pampas/ & [1pam.pas] \\
\hline papaya & /papaja/ & papaya & /papaja/ & [pa. ${ }^{1}$ paj.ja] \\
\hline peseta & /peseta/ & peseta & /peseta/ & [pe.1 $1_{\text {se:.ta] }}$ \\
\hline peso & /peso/ & peso & /pesu/ & [1pe:.su] \\
\hline picador & /pikador/ & pikador & /pikador/ & [pi.ka.1dor] \\
\hline pimiento & /pimmjento/ & pimiento & /pimientu/ & [pi.mi.1en.tu] \\
\hline platina & /platina/ & platina & /platina/ & [pla.1ti:.na] \\
\hline poncho & /pont5o/ & poncho & /pontşu/ & [1pon.tsu] \\
\hline quechua & /ketJwa/ & quechua & /ketşa/ & [1kct.su.a] \\
\hline rodeo & /rodeo/ & rodeo & /rudeu/ & [ru.1de:.u] \\
\hline rumba & /rumba/ & rumba & /rumba/ & {$\left[1_{\text {cum.ba }}\right]$} \\
\hline salsa & /salsa/ & salsa & /salsa/ & {$\left[1_{\text {sal.sa }}\right]$} \\
\hline sambo & /sambo/ & sambo & /sambu/ & {$\left[1_{\text {sam.bu }}\right]$} \\
\hline sangría & /sangria/ & sangria & /sangria/ & [say.2gri:a] \\
\hline siesta & /sjesta/ & siesta & /siesta/ & {$\left[\mathrm{si}^{1} \varepsilon \mathrm{s} . \mathrm{ta}\right]$} \\
\hline silo & /silo/ & silo & /silu/ & [1,si:.lu] \\
\hline sombrero & /sombrero/ & sombrero & /sumbreru/ & [sum.1bre:.su] \\
\hline taco & /tako/ & taco & /taku/ & [1ta:.ku] \\
\hline $\operatorname{tapa}(\mathrm{s})$ & $/ \operatorname{tapa}(\mathrm{s}) /$ & tapas & /tapas/ & [1ta:.pas] \\
\hline tapir & /tapir/ & tapir & /tapir/ & [ta.1pi:r] \\
\hline tilde & /tilde/ & tilde & /tilde/ & [2til.də] \\
\hline tortilla & $\begin{array}{l}\text { /tortiKa/ } \\
\text { /tortija/ }\end{array}$ & tortilla & $\begin{array}{l}\text { /tutija/ } \\
\text { /tutila/ }\end{array}$ & u. ${ }^{1}$ tij.ja] [tu. ${ }^{1}$ til.la] \\
\hline
\end{tabular}

